\title{
What's New in Neuraxial Labor Analgesia
}

\author{
Roulhac D. Toledano ${ }^{1} \cdot$ Lisa Leffert ${ }^{2,3}$
}

Accepted: 31 May 2021 / Published online: 27 August 2021

(C) Springer Science+Business Media, LLC, part of Springer Nature 2021

\begin{abstract}
Purpose of Review This article provides an update of recent practice trends in neuraxial labor analgesia. It reviews available evidence regarding management of labor pain in obstetric patients with COVID-19, serious adverse events in obstetric anesthesia to help inform risk/benefit decisions, and increasingly popular neuraxial labor analgesia techniques and adjuvants. State-of-theart modes of epidural drug delivery are also discussed.

Recent Findings There has recently been a focus on several considerations specific to obstetric anesthesia, such as anesthetic management of obstetric patients with COVID-19, platelet thresholds for the safe performance of neuraxial analgesia in obstetric patients with thrombocytopenia, and drug delivery modes for initiation and maintenance of neuraxial labor analgesia.

Summary Neuraxial labor analgesia (via standard epidural, dural puncture epidural, and combined spinal epidural techniques) is the most effective therapy to alleviate the pain of childbirth. SARS-CoV-2 infection is not, in and of itself, a contraindication to neuraxial labor analgesia or cesarean delivery anesthesia. Early initiation of neuraxial labor analgesia in patients with COVID-19 is recommended if not otherwise contraindicated, as it may reduce the need for general anesthesia should emergency cesarean delivery become necessary. Consensus regarding platelet thresholds for safe initiation of neuraxial procedures has historically been lacking. Recent studies have concluded that the risk of spinal epidural hematoma formation after neuraxial procedures is likely low at or above an imprecise range of platelet count of 70-75,000 $\times 10^{6} / \mathrm{L}$. Thrombocytopenia has been reported in obstetric patients with COVID-19, but severe thrombocytopenia precluding initiation of neuraxial anesthesia is extremely rare. High neuraxial blockade has emerged as one of the most common serious complications of neuraxial analgesia and anesthesia in obstetric patients. Growing awareness of factors that contribute to failed conversion of epidural labor analgesia to cesarean delivery anesthesia may help avoid the risks associated with performance of repeat neuraxial techniques and induction of general anesthesia after failed epidural blockade. Dural puncture techniques to alleviate the pain of childbirth continue to become more popular, as do adjuvant drugs to enhance or prolong neuraxial analgesia. Novel techniques for epidural drug delivery have become more widely disseminated.
\end{abstract}

Keywords COVID-19 $\cdot$ Anesthesia and analgesia $\cdot$ Neuraxial anesthesia $\cdot$ Epidural analgesia $\cdot$ Dural puncture epidural Single-shot spinal · Postpartum hemorrhage $\cdot$ Combined spinal epidural $\cdot$ Patient-controlled epidural analgesia $\cdot$ Programmed intermittent epidural bolus - Sterile precautions - Powered air-purifying respirator · Venous thromboembolism prophylaxis · Wire-reinforced epidural catheter $\cdot$ Accidental dural puncture $\cdot$ Postdural puncture headache $\cdot$ Epidural blood patch $\cdot$ Chronic headache $\cdot$ Immune thrombocytopenic purpura $\cdot$ Immune thrombocytopenia $\cdot$ Gestational thrombocytopenia $\cdot$ Hypertensive disorders of pregnancy · HELLP syndrome - Infectious complications - Bacterial meningitis - Spinal epidural hematoma · Platelet count · Platelet threshold $\cdot$ Spinal epidural abscess · Ultrasonography $\cdot$ Spinal cord injury $\cdot$ Peripheral $\cdot$ Failed epidural conversion $\cdot$ High neuraxial block

This article is part of the Topical Collection on Obstetric Anesthesia

Lisa Leffert

lisaleffert@gmail.com

1 NYU Langone Health, Department of Anesthesiology, Perioperative Care \& Pain Medicine, NYU Langone Hospital, Brooklyn, USA

2 Department of Anesthesia, Critical Care \& Pain Medicine, Harvard Medical School, Boston, USA

3 Obstetric Anesthesia Division, Massachusetts General Hospital, Boston, MA, USA

\section{Introduction}

Single-shot spinal, standard epidural, combined spinal epidural (CSE), and dural puncture epidural (DPE) are the most effective techniques to alleviate the pain of childbirth. Initiation of neuraxial blockade in laboring patients provides reliable and rapid onset of high-quality pain relief with minimal serious side effects to the mother and fetus. Catheterbased techniques (epidural, CSE, DPE) also provide a means 
of rapid conversion to surgical anesthesia for operative delivery. In situ catheters that can be utilized successfully for cesarean delivery anesthesia minimize the need for induction of general anesthesia (GA) and thereby reduce the likelihood of exposure to the risks of GA in this patient population (Table 1).

This article provides an up-to-date, evidence-based review of current trends in neuraxial labor analgesia, including strategies for optimizing and customizing analgesia. We will also include a discussion of how to navigate providing neuraxial labor analgesia during the COVID-19 pandemic.

\section{Preparation for Neuraxial Procedures}

While our understanding of COVID-19 is still evolving, the pandemic has changed several aspects of the management of obstetric patients. Preparation for a neuraxial procedure now requires an understanding of how to screen and prepare for patients with SARS-CoV-2 infection.

\section{Preparation for Neuraxial Labor Analgesia during the COVID-19 Pandemic}

All patients should be screened for COVID-19 symptoms remotely (i.e., by phone or video) before entrance to the labor and delivery floor. Ideally, universal testing should be employed so that the SARS-CoV-2 status of all hospitalized patients is known. Healthcare providers should use contact and droplet precautions with eye protection (gown, gloves, surgical mask, face shield) when in contact with a patient with known or suspected COVID-19 and when undertaking neuraxial procedures for labor, in accordance with several obstetric anesthesia society recommendations. Airborne precautions with an N95 mask and face shield (or a powered airpurifying respirator (PAPR) if these have an insufficient seal)

Table 1 Adverse events associated with general anesthesia in obstetric patients should be used during aerosol-generating procedures such as intubation [1]. Equipment in rooms should be limited to necessary drugs and labor analgesia and intubating supplies ("COVID kits") to avoid contamination of anesthesia carts and drug-dispensing machines. If feasible, it is also prudent to limit the number of healthcare providers caring for COVID19 patients; a log should be maintained of every staff member who goes in and out of the labor and operating rooms.

Early neuraxial labor analgesia is recommended for obstetric patients with COVID-19 unless otherwise contraindicated, as it may reduce the need for GA should emergency cesarean delivery become necessary. Because patients with COVID-19 may require venous thromboembolism (VTE) prophylaxis, consideration of the timing and dose of the last anticoagulation medication may be required [2••]. It is also advisable to check a platelet count prior to initiation of neuraxial procedures, although thrombocytopenia is typically only reported in patients with severe COVID-19 illness. However, severe thrombocytopenia in laboring patients that precludes neuraxial analgesia and anesthesia is rare, except in the presence of other etiologies of thrombocytopenia, disseminated intravascular coagulation (DIC), additional comorbidities, or severe disease.

\section{Informed Consent}

Neuraxial procedures in obstetric patients have a strong safety record, but mild, transient complications and rare, serious, life-threatening complications can occur. Postdural puncture headache (PDPH) is a common, potentially severe complication of neuraxial labor procedures, with an incidence of roughly $0.7-1 \%[3,4]$. PDPH develops in an estimated $52-60 \%$ of obstetric patients after accidental dural puncture (ADP) with an epidural needle and is far less frequent after spinal techniques (0.5-2\% with small pencil-point spinal needles) [5]. Recent studies suggest that PDPH is, rarely, associated with increased risk of major neurologic complications (e.g., cerebral venous thrombosis, subdural hematoma, bacterial meningitis, persistent low back pain, chronic headache) [6•].

High neuraxial blockade has been identified as one of the most common serious complications of neuraxial anesthesia and analgesia in obstetric patients, with an incidence of roughly $1 / 4336$ neuraxial procedures [3]. This adverse event most often occurs in the labor suite as a result of unrecognized spinal catheter placement during labor epidural procedures but can also occur when spinal anesthesia for surgical delivery is administered after a failed epidural anesthetic (which may complicate up to $14 \%$ of epidural placements) [7]. It is reasonable to discuss with patients that optimal placement of the epidural catheter for labor analgesia and/or surgical anesthesia cannot be guaranteed and that the catheter may require replacement if pain relief is partial or failed or should cesarean delivery become necessary. Growing awareness of factors that 
contribute to failed labor epidural conversion to cesarean delivery anesthesia (Table 2), as well as active management of in situ epidural catheters and early replacement of nonfunctioning catheters, may help reduce the incidence of high neuraxial blockade and limit the use of GA in obstetrics $[8,9]$.

Given the rarity of serious neurologic complications of neuraxial blockade in obstetric patients, accurate estimates of the incidence of such events are difficult to gauge. Infection (meningitis, spinal epidural abscess) is a rare cause of serious neurologic sequelae [10]. The risk of direct spinal cord or permanent nerve root damage is extremely low in obstetric patients. To try to minimize trauma during neuraxial procedures, we require that patients report any pain or paresthesias that may occur during the neuraxial procedure and then move the needle or catheter accordingly. In addition, ultrasound can assist in more accurately identifying the lower lumbar interspaces (e.g., L4,5 or L3,4) that are more likely to be below the conus medullaris (the tapered, lower end of the spinal cord which typically occurs near lumber vertebral levels 1 and 2 but may be lower).

Spinal epidural hematoma (SEH), the most feared complication of neuraxial anesthesia, has traditionally been associated with anticoagulation therapy, severe thrombocytopenia, traumatic procedures requiring multiple attempts, existing spinal pathology (e.g., spinal stenosis), and thoracic procedures in non-obstetric patients [11]. Obstetric patients are at a lower risk for SEH formation when compared with the general population, with an estimated incidence of 1:200,000-1:250,000 versus 1:3600 in the elderly orthopedic female [11]. Potential reasons include a compliant epidural space (especially in the lumbar region where neuraxial labor analgesia is performed), the hypercoagulable state of pregnancy, a relatively low incidence of spinal pathology, and a lower likelihood of therapeutic anticoagulation.

Thrombocytopenia affects up to $12 \%$ of obstetric patients, with $\sim 1 \%$ having a platelet count $<100,000 \times 10^{6} / \mathrm{L}$. [12] High-quality data to guide anesthesia providers about whether

Table 2 Factors associated with failed epidural conversion (*validated in a systematic review and meta-analysis: Bauer ME, Kountanis JA, Tsen LC, Greenfield ML, Mhyre JM. Int J Obstet Anesth 2012;21:294 309)

\section{Placement by a non-obstetric anesthesiologist/nonspecialist* \\ Performance of a standard epidural technique (compared with CSE, DPE) \\ Increasing number of clinician-administered boluses for breakthrough pain* \\ Increased urgency for cesarean delivery* Prolonged duration of analgesia Higher body mass index Placement in early labor}

CSE combined spinal epidural procedure, $D P E$ dural puncture epidural procedure to proceed with neuraxial techniques in thrombocytopenic obstetric patients are limited. A recent meta-analysis reporting lumbar neuraxial procedures (i.e., lumbar puncture; spinal, epidural, CSE procedures; epidural catheter removal) in thrombocytopenic patients (defined as platelet count $<$ $100,000 \times 10^{6} / \mathrm{L}$ ) across populations (e.g. pediatric and adult oncology patients, obstetric patients) concluded that SEH is rare $[13 \cdot \bullet]$. Thirty-three cases of SEH were identified from a total of 7476 procedures between the years 1947 and 2018, most commonly after lumbar punctures $(75.8 \%)$ with platelet counts $<50,000 \times 10^{6} / \mathrm{L}(61 \%)$. Five cases of SEH in obstetric patients with thrombocytopenia were reported: one in a patient who was coagulopathic at the time of accidental epidural catheter removal, one who had an underlying spinal arteriovenous malformation (AVM), two with hemolysis, elevated liver enzymes, and low platelets (HELLP) syndrome; and one with eclampsia. The authors concluded that the sample probability of spinal epidural hematoma formation for all neuraxial procedures is likely low, above an imprecise range of platelet count $\geq 70-75,000 \times 10^{6} / \mathrm{L}[0.097 \%$ (95\% CI $\left.0.002,0.2 \%)\right]$.

Subsequently, a Society for Obstetric Anesthesia and Perinatology (SOAP) interdisciplinary consensus statement on neuraxial procedures in thrombocytopenic obstetric patients concluded that if the platelet count is $\geq 70,000 \times 10^{6} / \mathrm{L}$ and there are no additional contraindications or risk factors, then there is likely to be a low risk of spinal epidural hemato$\mathrm{ma}$ and it is reasonable to proceed with a neuraxial procedure if clinically indicated (class IIa and level C-LD) [2••]. In the subgroup with a known etiology of thrombocytopenia and a platelet count between 50,000 and $70,000 \times 10^{6} / \mathrm{L}$, an individualized risk/benefit analysis within the clinical context is required to determine the appropriateness of neuraxial anesthesia. If the platelet count is below $50,000 \times 10^{6} / \mathrm{L}$, then there may likely be an increased risk of SEH, and it may be reasonable to avoid neuraxial procedures (class IIb and level C-LD). Obstetric patients who present to the labor and delivery floor with newly recognized thrombocytopenia may require additional work-up before initiation of neuraxial labor analgesia if the platelet count is $<70,000$ $\times 10^{6} / \mathrm{L}$. The SOAP consensus statement is not intended to establish a standard of care or to replace medical judgement but rather to provide guidance for weighing the relative risks and benefits of performing neuraxial procedures in obstetric patients with thrombocytopenia.

\section{Physical Examination and Laboratory Studies}

A focused history and physical examination are recommended prior to initiation of neuraxial labor analgesia. The American Society of Anesthesiology (ASA) and SOAP practice guidelines for obstetric anesthesia endorse that a platelet count is not required prior to initiation of neuraxial blockade in low-risk obstetric patients [14]. Rather, the anesthesiologist's decision 
to order a platelet count should be individualized based on the patient's obstetric history (e.g., HELLP (hemolysis, elevated liver enzymes, and low platelet count syndrome)) a history of clinically concerning mucocutaneous bleeding or petechiae, or other comorbid states (e.g., severe thrombocytopenia without a known etiology). Severe thrombocytopenia and a change in platelet count from $>100,000 \times 10^{6} / \mathrm{L}$ to $<100,000 \times 10^{6} / \mathrm{L}$ was found to be extremely rare in a recent retrospective review of 984 patients with pre-eclampsia, except in the case of patients with HELLP syndrome [15•].

In general, obstetric patients are hypercoagulable due to an increase in most procoagulant factors and most commonly have normal hemostasis parameters. When evaluating patients for neuraxial labor analgesia, the PT and aPTT may be most clinically useful when patients are on exogenous anticoagulants and have DIC or other inherited or acquired coagulation defects.

There are some reports of thromboelastography (TEG) and rotational thromboelastometry (ROTEM) being used to predict bleeding risk prior to initiation of neuraxial anesthesia and analgesia, including in thrombocytopenic obstetric patients. However, the correlation between TEG and ROTEM parameters and clinical bleeding may be poor, except at very low platelet counts [16]. As such, there is insufficient evidence to recommend the routine use of viscoelastic point of care testing in thrombocytopenic obstetric patients, including those with preeclampsia, to determine the safety of neuraxial techniques [2••].

\section{Equipment for Neuraxial Blockade}

Several advances in epidural catheter design and epidural drug delivery technology have improved the efficacy of neuraxial labor analgesia over the past decades.

\section{Epidural Catheter Design}

Epidural catheters are available in nylon blends with intermediate bending stiffness and in more flexible, wire-reinforced versions with either a nylon or polyurethane coating around the inner spring-wound coil. They also are made in single-end hole or multi-orifice designs. Some traditional nylon catheters have flexible tips to minimize the risks described below that have been associated with stiffer catheters.

Several studies have demonstrated a lower incidence of epidural vein cannulation, paresthesia, and catheter migration with the use of flexible wire-reinforced catheters when compared with stiffer nylon versions [17]. However, wirereinforced catheters can be harder to thread, likely by virtue of the flexibility afforded by the stainless-steel wire coil and the material properties of the polyurethane or nylon blend coating surrounding the inner coil. If there is difficulty threading the catheter, then consider distending the epidural space with additional saline, retracting the epidural needle and

re-identifying the epidural space, using a stiffer nylon catheter in lieu of a flexible catheter, or repeating the procedure at a different interspace [18] (Table 3). Do not separately withdraw a catheter that has already exited the tip of the needle; take the needle and catheter out together to avoid catheter sheering. Wire-reinforced catheters have limited magnetic resonance imaging (MRI) compatibility, which may be important if obstetric patients require MRI peripartum neurologic evaluation; some are considered "magnetic resonance-conditional," which allows for use under specific conditions [17]. There appears to be no difference in clinical outcomes (e.g., analgesic efficacy, episodes of breakthrough pain, occurrence of complications) between single-end hole catheters and multiorifice designs when wire-reinforced catheters are used [19]. We use single-end hole wire-reinforced polyurethane catheters with good effect.

\section{Spinal Needle Type and Gauge for CSE and DPE Techniques}

CSE kits are available with either a 25-, 26-, or 27-gauge long spinal needle. Alternatively, a 5-in. $(12.7 \mathrm{~cm})$ spinal needle that is compatible with the epidural needle provided in your epidural kit can be added to the sterile field. Epidural manufacturers and distributors in the USA provide different needle designs of a specific gauge in their non-custom CSE kits. Before performing a dural puncture technique, it is important to confirm that the spinal and epidural needle pair that you are using is compatible; the spinal needle must exit the epidural needle with sufficient length to reach the dura.

\section{Drug Delivery Techniques}

Pump infusion technology has evolved over the past decades. Patient-controlled epidural anesthesia (PCEA) has largely replaced continuous epidural infusion (CEI) technology. With PCEA, a background continuous infusion is most commonly provided, with self-administered patient boluses at

Table 3 Corrective maneuvers to facilitate advancing flexible wirereinforced catheters*

\footnotetext{
Administer saline through epidural needle and retry

Identify epidural space at a different vertebral level

Withdraw epidural needle, re-engage in ligament, and re-advance

Use a stiffer catheter

Change angle of needle slightly (note: rotating the needle has been associated with an increased in accidental dural puncture)
}

*Do not remove catheter separately from the needle, once it has been advanced at all through the tip of the epidural needle 
predetermined time intervals. PCEA with continuous background infusion may reduce the need for clinicianadministered boluses and improve maternal analgesia [20], but may increase the incidence of motor block, depending on the concentration of local anesthetic (LA) [21]. Optimal PCEA settings have not been determined, but common patient bolus regimens with varying background infusions include 8$10 \mathrm{~mL} 0.0625 \%$ bupivacaine with $2 \mu / \mathrm{mL}$ fentanyl every 10 $\min ; 6-8 \mathrm{~mL} 0.08-0.1 \%$ bupivacaine with $2 \mu / \mathrm{mL}$ fentanyl every $15 \mathrm{~min}$; or $10 \mathrm{~mL} 0.1 \%$ ropivacaine with $2 \mu / \mathrm{mL}$ fentanyl every $10-15 \mathrm{~min}$. In order to optimize maternal satisfaction, it is important to address patient expectations and training when using the PCEA technique. New PCEA technologies, including disposable devices and computer-integrated infusion pumps which modify the background infusion based on patient bolus requirements, are being developed.

More recently, patient-controlled intermittent bolus (PIEB) techniques for the administration of epidural infusion medications have replaced continuous infusion and PCEA techniques at many institutions. With PIEB, the pump itself administers high-pressure boluses at predetermined intervals. Most PIEB technology permits patient-controlled boluses in addition to the programmed pump boluses. The PIEB technique has been shown in several studies to improve patient comfort and satisfaction, reduce total LA consumption, and reduce clinicianadministered boluses without increasing the incidence of motor block [22]. Rare cases of high block after inadvertent intrathecal catheter placement or unrecognized catheter migration have been reported in the literature. Determining the optimal dose and time interval for programmed boluses with epidural solutions of different concentrations has proven challenging. Bupivacaine $0.0625 \%$ with fentanyl $2 \mu, 5 \mathrm{~mL}$ PCEA boluses, $6 \mathrm{~mL}$ PIEB q $30 \mathrm{~min}$ [23] and ropivacaine $0.1 \%$ with fentanyl $2 \mu / \mathrm{mL}, 5 \mathrm{~mL}$ PCEA boluses, $5 \mathrm{~mL}$ PIEB q $1 \mathrm{~h}$ [24] are among several options for a PIEB technique.

\section{Neuraxial Ultrasound}

Ultrasound identification of the intended lumbar interspace and estimation of the depth to epidural space is being used increasingly in patients with challenging anatomy (e.g., parturients with obesity or scoliosis), as well as for routine epidural placements. A recent systematic review (31 clinical trials and 1 meta-analysis) concluded that neuraxial ultrasound was more accurate than palpation in identifying a specific lumbar interspace and provided an excellent estimate of depth to the epidural or intrathecal space [25]. In addition, it showed that ultrasound increased the overall success and reduced the risk of traumatic procedures in a manner superior to palpation. Because becoming proficient in neuraxial use in patients with complex anatomy requires facility with the tool, we advocate the routine use of ultrasound, when feasible, prior to initiation of neuraxial blockade in obstetric patients.

\section{Neuraxial Labor Analgesia Techniques}

Neuraxial labor analgesia can be initiated with a single-shot spinal, standard epidural, CSE, or DPE technique, depending on patient- and provider-specific factors.

\section{Single-Shot Spinal Technique}

A single-shot spinal technique may be appropriate if delivery is imminent or when a catheter-based technique is not feasible. Adding adjuvants (e.g., epinephrine) [26] to the spinal medication can prolong the effect. If labor is unexpectedly prolonged, an epidural catheter or repeat single-shot spinal procedure may be required. Unlike an epidural or CSE neuraxial analgesic technique, single-shot spinal labor analgesia does not provide an in situ catheter which can then be used to provide surgical anesthesia for unplanned cesarean delivery or for postpartum bleeding complications and procedures.

\section{Standard Epidural Technique}

The standard epidural technique is a common neuraxial procedure for labor analgesia as it is easy to perform and provides effective analgesia. After the epidural space is identified with the loss of resistance (LOR) technique and the epidural catheter is threaded no more than $6 \mathrm{~cm}$ into the space [27], a loading dose of LA with or without an opioid (most commonly with) is administered in $3-5 \mathrm{~mL}$ aliquots over several minutes, with intermittent blood pressure (BP) and heart rate (HR) assessments.

Other adjuvants can be added to the dilute LA solutions in current clinical use (bupivacaine $0.0625-0.1 \%$ or ropivacaine $0.08-0.1 \%$ ) to improve analgesia, reduce LA consumption, and prolong the analgesic effect. The addition of dexmedetomidine $(0.5 \mu \mathrm{g} / \mathrm{mL})$, an $\alpha 2$-adrenoreceptor agonist, to LA epidural solutions has been shown to provide comparable or superior labor analgesia with fewer side effects (e.g., pruritus, nausea, and vomiting) when compared with opioid adjuvants (sufentanil) [28]. Studies are also evaluating the safety, efficacy, optimal dose, and mode of drug delivery (i.e., PIEB, PCEA) with clonidine, an $\alpha 2$-agonist, and/or neostigmine, an acetylcholinesterase inhibitor, adjuvants to the LA solution, with or without opioid [29].

The standard epidural technique requires larger doses of LA to initiate analgesia (i.e., the loading dose of up to 20 $\mathrm{mL}$ of dilute epidural solution). An epinephrine-containing test dose can be administered to help identify inadvertent intrathecal or intravenous catheter placement if used as the first part of the loading dose. If a misplaced catheter is not properly identified, a high or total neuraxial blockade or local anesthetic systemic toxicity (LAST) can ensue. 


\section{Combined Spinal Epidural Technique}

The CSE technique is used increasingly by obstetric anesthesiologists and other providers with specialized training in the field. After the epidural space is identified via the loss of resistance technique, a 25-, 26-, or 27-gauge spinal needle is advanced via the epidural needle through the dura into the subarachnoid space. An opioid (e.g., fentanyl, 10-20 $\mu$ )), most commonly in combination with an LA (e.g., bupivacaine $1-2.5 \mathrm{mg}$ ), is administered into the subarachnoid space before the spinal needle is withdrawn and the epidural catheter is threaded into the epidural space. Dexmedetomidine $(10 \mu \mathrm{g})$ can be administered, in lieu of an opioid, for the intrathecal component [30]. Intrathecal neostigmine $(50 \mu \mathrm{g})$ and clonidine $(75 \mu \mathrm{g})$ have also been used to augment analgesia in nonobstetric patients in one study with no adverse effects [31].

Potential benefits of the CSE technique, when compared with the standard epidural technique, include faster onset of analgesia (3-5 min versus $20 \mathrm{~min}$ ), higher maternal satisfaction, lower incidence of unilateral or patchy block, reduced need for rescue analgesia, reduced total drug dosage, improved sacral coverage [32], and increased maternal mobility [33]. The use of a CSE technique instead of a standard epidural is one of the several factors associated with the successful conversion of labor analgesia to cesarean delivery anesthesia. The CSE technique has also been associated with more rapid cervical dilation in one study when compared with epidural analgesia in nulliparous women in early labor [34].

The CSE technique is associated with an increased risk of fetal bradycardia [35] (not associated with an increased risk of cesarean delivery) and opioid-induced pruritus. Fetal bradycardia is postulated to result in part from opioid-induced uterine hypertonus and may be avoided by reducing the dose of intrathecal opioid [36]. Non-reassuring fetal heart rate after CSE labor analgesia can most often be treated with several commonly performed maneuvers, including a change in maternal position, fluid and terbutaline administration, and an intravenous dose of ephedrine or phenylephrine. Ultimately, there appears to be no difference in mode of delivery between CSE and standard epidural analgesic techniques [37]. Pruritus after CSE usually resolves after 45-60 $\mathrm{min}$ and can be minimized by decreasing the dose of fentanyl (to $10-15 \mathrm{mcg}$ ). A small intravenous dose (i.e., $5 \mathrm{mg}$ ) of the mixed agonist-antagonist nalbuphine successfully reverses pruritus without reversing analgesia. Concerns for increased risk of infection or PDPH related to the dural puncture have not been substantiated in the literature.

Although the functionality of epidural catheters placed during a CSE technique is not known at the outset of the block, evidence suggests that these catheters are as or more reliable than epidural catheter placed during standard epidural procedures.

\section{Dural Puncture Epidural Technique}

The DPE technique for labor analgesia has become increasingly popular for labor analgesia. The procedure is similar to the CSE technique, except that no intrathecal medication is administered. Studies of DPE techniques are equivocal as to the extent to which the dural puncture epidural is superior to the traditional epidural technique. One recent systematic review concluded that there was a lack of clear benefit over traditional epidural techniques [38•]. A second noted that although the "collective results remain ambiguous," 25-gauge (but not 26- or 27gauge) spinal needles have been reported to provide higher success rates than standard epidural techniques without dural puncture [39]. Two other studies agreed that dural puncture epidural results in fewer unilateral blocks and better sacral coverage than traditional epidural analgesia.

When compared with the CSE technique, the DPE technique results in comparable analgesia in a somewhat comparable timeframe (median 2 min versus $11 \mathrm{~min}$ ) with fewer maternal and fetal side effects (e.g., maternal pruritus, hypotension, fetal bradycardia) [40]. Both techniques can be used to aid with epidural space localization when LOR is equivocal or difficult to discern. CSF flow through the spinal needle provides supportive evidence that the tip of the epidural needle is close to the dura and therefore likely in the epidural space.

\section{Conclusion}

Neuraxial labor analgesia continues to be the technique that provides optimal and versatile pain management during labor and the potential for conversion to an anesthetic for cesarean delivery if needed. Procedural techniques such as CSE and DPE have shown promise in improving sacral coverage and reducing the number of unilateral blocks. An increasing number of adjuncts to local anesthetics are being explored to augment the quality and duration of the block. Finally, drug delivery systems (e.g., PIEB) continue to be refined to capitalize on the advantage of bolus dosing to best pair patient need with drug delivery.

\section{Declaration}

Conflict of Interest The authors do not have any potential conflicts of interest to disclose 


\section{References}

Papers of particular interest, published recently, have been highlighted as:

- Of importance

•- Of major importance

1. www.SOAP.org.

2.• Bauer MA, Beilin K, Gernsheimer Y, T Perez Botero J, James AH, Yaghmour E, et al. The Society for Obstetric Anesthesia and Perinatology Interdisciplinary Consesnsus Statement on Neuraxial Procedures in Obstetric Patients with Thrombocytopenia. Anesth Analg. 2021; This recent interdisciplinary consensus statement focuses on the considerations and decisions which need to be made as an anesthesiologist performing neuraxial procedures for obstetric patients with thrombocytopenia. It provides recommendations and decision-making support.

3. D'Angelo R, Smiley RM, Riley ET, Segal S. Serious complications related to obstetric anesthesia: the serious complication repository project of the Society for Obstetric Anesthesia and Perinatology. Anesthesiology. 2014;120(6):1505-12.

4. Sachs A, Smiley R. Post-dural puncture headache: the worst common complication in obstetric anesthesia. Semin Perinatol. 2014;38(6):386-94.

5. Orbach-Zinger S, Ashwal E, Hazan L, Bracco D, Ioscovich A, Hiersch L, et al. Risk factors for unintended dural puncture in obstetric patients: a retrospective cohort study. Anesth Analg. 2016;123(4):972-6.

6. Guglielminotti J, Landau R, Li G. Major neurologic complications associated with postdural puncture headache in obstetrics: a retrospective cohort study. Anesth Analg. 2019;129(5):1328-36 This retrospective cohort study included over $1,000,000$ million women who received neuraxial anesthesia for labor in New York State between 2005 and 2014. PDPH was found to be associated with major neurologic complications.

7. Pan PH, Bogard TD, Owen MD. Incidence and characteristics of failures in obstetric neuraxial analgesia and anesthesia: a retrospective analysis of 19,259 deliveries. Int J Obstet Anesth. 2004;13(4): 227-33.

8. Bauer ME, Kountanis JA, Tsen LC, Greenfield ML, Mhyre JM. Risk factors for failed conversion of labor epidural analgesia to cesarean delivery anesthesia: a systematic review and metaanalysis of observational trials. Int J Obstet Anesth. 2012;21(4): 294-309.

9. Mankowitz SK, Gonzalez Fiol A, Smiley R. Failure to extend epidural labor analgesia for cesarean delivery anesthesia: a focused review. Anesth Analg. 2016;123(5):1174-80.

10. Toledano RW, D. Serious neurologic complications of neuraxial anesthesia procedures in obstetric patients. UpToDate. 2020.

11. Moen V, Dahlgren N, Irestedt L. Severe neurological complications after central neuraxial blockades in Sweden 1990-1999. Anesthesiology. 2004;101(4):950-9.

12. Reese JA, Peck JD, Deschamps DR, McIntosh JJ, Knudtson EJ, Terrell DR, et al. Platelet counts during pregnancy. $N$ Engl J Med. 2018;379(1):32-43.

13.• Bauer ME, Toledano RD, Houle T, et al. Lumbar neuraxial procedures in thrombocytopenic patients across populations: A systematic review and meta-analysis. J Clin Anesth. 2020;61:109666 This systematic review and meta-analysis compares and evaluates lumbar neuraxial procedures in thrombocytopenic patients across many patients populations.

14. Practice Guidelines for Obstetric Anesthesia. An updated report by the American Society of Anesthesiologists Task Force on Obstetric
Anesthesia and the Society for obstetric anesthesia and perinatology. Anesthesiology. 2016;124(2):270-300.

15. Beilin Y, Katz DJ. Analgesia use among 984 women with preeclampsia: A retrospective observational single-center study. $J$ Clin Anesth. 2020;62:109741 This retrospective, observational study investigates the incidence of thrombocytopenia and platelet count trends in the $\mathbf{7 2} \mathrm{h}$ before delivery among women with preeclampsia.

16. Kim SY, Gu JY, Yoo HJ, Kim JE, Jang S, Choe S, et al. Benefits of thromboelastography and thrombin generation assay for bleeding prediction in patients with thrombocytopenia or hematologic malignancies. Annals of laboratory medicine. 2017;37(6):484-93.

17. Toledano RD, Tsen LC. Epidural catheter design: history, innovations, and clinical implications. Anesthesiology. 2014;121(1):9-17.

18. Sviggum HP, Farber MK. The incidence and management of inability to advance Arrow FlexTip Plus epidural catheters in obstetric patients. Int J Obstet Anesth. 2014;23(2):113-7.

19. Spiegel JE, Vasudevan A, Li Y, Hess PE. A randomized prospective study comparing two flexible epidural catheters for labour analgesia. Br J Anaesth. 2009;103(3):400-5.

20. Halpern SH, Carvalho B. Patient-controlled epidural analgesia for labor. Anesth Analg. 2009;108(3):921-8.

21. van der Vyver M, Halpern S, Joseph G. Patient-controlled epidural analgesia versus continuous infusion for labour analgesia: a metaanalysis. Br J Anaesth. 2002;89(3):459-65.

22. Hussain N, Lagnese, Hayes B, et al. Comparative analgesic efficacy and safety of intermittent local anaesthetic epidural bolus for labour: a systematic review and meta-analysis. Br J Anaesth. 2020;125(4): 560-79.

23. Wong CA, Ratliff JT, Sullivan JT, Scavone BM, Toledo P, McCarthy RJ. A randomized comparison of programmed intermittent epidural bolus with continuous epidural infusion for labor analgesia. Anesth Analg. 2006;102(3):904-9.

24. Bullingham A, Liang S, Edmonds E, Mathur S, Sharma S. Continuous epidural infusion vs programmed intermittent epidural bolus for labour analgesia: a prospective, controlled, before-andafter cohort study of labour outcomes. Br J Anaesth. 2018;121(2): $432-7$.

25. Perlas A, Chaparro LE, Chin KJ. Lumbar neuraxial ultrasound for spinal and epidural anesthesia: a systematic review and meta-analysis. Reg Anesth Pain Med. 2016;41(2):251-60.

26. Gurbet A, Turker G, Kose DO, Uckunkaya N. Intrathecal epinephrine in combined spinal-epidural analgesia for labor: dose-response relationship for epinephrine added to a local anesthetic-opioid combination. Int J Obstet Anesth. 2005;14(2):121-5.

27. Mhyre JM, Greenfield ML, Tsen LC, Polley LS. A systematic review of randomized controlled trials that evaluate strategies to avoid epidural vein cannulation during obstetric epidural catheter placement. Anesth Analg. 2009;108(4):1232-42.

28. Zhang T, Yu Y, Zhang W, Zhu J. Comparison of dexmedetomidine and sufentanil as adjuvants to local anesthetic for epidural labor analgesia: a randomized controlled trial. Drug design, development and therapy. 2019;13:1171-5.

29. Boogmans T, Vertommen J, Valkenborgh T, Devroe S, Roofthooft E, Van de Velde M. Epidural neostigmine and clonidine improves the quality of combined spinal epidural analgesia in labour: a randomised, double-blind controlled trial. Eur J Anaesthesiol. 2014;31(4):190-6.

30. Dilesh P, Eapen S, Kiran S, Chopra V. A comparison of intrathecal dexmedetomidine verses intrathecal fentanyl with epidural bupivacaine for combined spinal epidural labor analgesia. Journal of Obstetric Anaesthesia and Critical Care. 2014;4(2):69-74.

31. Yoganarasimha N, Raghavendra T, Amitha S, Shridhar K, Radha M. A comparative study between intrathecal clonidine and neostigmine with intrathecal bupivacaine for lower abdominal surgeries. Indian journal of anaesthesia. 2014;58(1):43-7. 
32. Cappiello E, O'Rourke N, Segal S, Tsen LC. A randomized trial of dural puncture epidural technique compared with the standard epidural technique for labor analgesia. Anesth Analg. 2008;107(5): 1646-51.

33. Simmons SW, Taghizadeh N, Dennis AT, Hughes D, Cyna AM. Combined spinal-epidural versus epidural analgesia in labour. The Cochrane database of systematic reviews. 2012;10(10):Cd003401.

34. Tsen LC, Thue B, Datta S, Segal S. Is combined spinal-epidural analgesia associated with more rapid cervical dilation in nulliparous patients when compared with conventional epidural analgesia? Anesthesiology. 1999;91(4):920-5.

35. Hattler J, Klimek M, Rossaint R, Heesen M. The effect of combined spinal-epidural versus epidural analgesia in laboring women on nonreassuring fetal heart rate tracings: systematic review and meta-analysis. Anesth Analg. 2016;123(4):955-64.

36. Friedlander JD, Fox HE, Cain CF, Dominguez CL, Smiley RM. Fetal bradycardia and uterine hyperactivity following subarachnoid administration of fentanyl during labor. Reg Anesth. 1997;22(4): $378-81$.
37. Collis RE, Davies DW, Aveling W. Randomised comparison of combined spinal-epidural and standard epidural analgesia in labour. Lancet (London, England). 1995;345(8962):1413-6.

38. Heesen M, Carvalho B, Carvalho JCA, et al. International consensus statement on the use of uterotonic agents during caesarean section. Anaesthesia. 2019;74(10):1305-19 This international consensus statement advises on the use and dosing of uterotonic agents during and following cesarean delivery.

39. Layera S, Bravo D, Aliste J, Tran DQ. A systematic review of DURAL puncture epidural analgesia for labor. J Clin Anesth. 2019;53:5-10.

40. Chau A, Bibbo C, Huang CC, Elterman KG, Cappiello EC, Robinson JN, et al. Dural puncture epidural technique improves labor analgesia quality with fewer side effects compared with epidural and combined spinal epidural techniques: a randomized clinical trial. Anesth Analg. 2017;124(2):560-9.

Publisher's Note Springer Nature remains neutral with regard to jurisdictional claims in published maps and institutional affiliations. 